\title{
A Model-Based Filtering Strategy to Reconstruct the Maximum Power Generation of Curtailed Photovoltaic Installations: application to forecasting
}

\author{
E. Scolari, F. Sossan, M. Paolone \\ École Polytechnique Fédérale de Lausanne, Switzerland
}

\begin{abstract}
In this paper, we propose a model-based filtering strategy to reconstruct the maximum power production of a $\mathbf{P V}$ power plant thanks to integrating measurements of the PV cell temperature, system DC voltage and DC current. The filter relies on a reversed physical model of the targeted PV system and enables to, first, determine analytically the irradiance incident to the panels, and, second, estimate the DC power production as if the plant was operating in maximum power point tracking (MPPT) mode. We present how the approach can be used to reconstruct the maximum power value starting from a generic operating point. As an application, we show that the proposed strategy can improve time series-based solar power forecasting techniques, in particular when the production of the PV system is curtailed and thus the measured power does not correspond to the maximum available.
\end{abstract}

Index Terms-Solar power estimation, maximum power forecast, Photovoltaic (PV) system, stochastic generation.

\section{INTRODUCTION}

$\mathbf{T}$ HE development of robust control strategies based on PV generation forecasts is envisaged as a key practice to mitigate the operational issues related to its increased diffusion in power distribution networks. In particular, when considering stochastic generation (e.g. PVs), it is desirable to predict the maximum power that will be available in the coming forecast horizon and to estimate the associated uncertainty. This is important, for example, to adequately plan counteractions to avoid operational issues and to schedule the reserves, [1].

Two approaches are normally possible to forecast PV power generation: indirect and direct methods [2]. The former consists in coupling prediction models of the irradiance, see [3], with physical models of the PV system. The latter consists in applying statistical and machine learning methods trained on historical PV production time series assumed to be representative of the typical variations patterns of the irradiance, or, in other words, of the PV system maximum power. They recently come to prominence to tackle the problem of shortterm forecasting at a large disaggregation level, also thanks to the progressively increasing availability of measurements of local PV generation, e.g. [4], [5]. Although the use of locally available information is appealing, the argument on using direct forecasting methodologies might fall flat if considering that production measurements might be altered by curtailment strategies, like when PV plants are required to support voltage or primary/secondary frequency regulation, e.g. [6]. In this case, existing direct forecasting methods should be augmented to consider the fact that PV plants might not operate in the typical maximum power point tracking (MPPT) mode.

In this paper, we propose a model-based filtering strategy to reconstruct the maximum production profile of a PV power plant starting from generic conditions (which might include curtailed regimes) thanks to integrating measurements of the PV cell temperature $T$, DC voltage $v$ and DC current $i$. The filter relies on a reversed physical model of the targeted PV array and enables to analytically determine the irradiance incident on the panel, and, then, to estimate the DC power production as if the plant was operating in MPPT mode. The reverse physical model adopted in the filtering strategy is developed from the five-parameter cell model of De Soto et al. in [7], extended to the whole PV array. This model has the main advantage of requiring only datasheet information to fit the parameters. Furthermore, the proposed reverse approach allows to avoid irradiance sensors, which are expensive, can have an absorption spectrum different from the PV panel, and return local measurements that are not representative of the irradiance on the whole plant (e.g. when part of the plant is shaded or only partially covered by clouds). The performance of the proposed algorithm is evaluated by first estimating the maximum power, starting from the measured $T, v$ and $i$, and considering the converter working both in MPPT regime and curtailed one. Then, the estimation is compared with the true maximum power value, assumed as the one returned by a second identical converter, working under same conditions, and always operating in MPPT mode. Finally, as an application of the method, we show how generic direct forecasting tools of PV power might benefit from the proposed filtering strategy and improve their performance, compared to the case where past row power measurements are directly used to feed the algorithm. The paper is organized as follows: Section II briefly introduces the state-of-the-art while Section $\amalg$ states the main problem tackled in the work. Section IV describes in details the proposed approach. Section $\mathrm{V}$ illustrates the experimental setup while Section VI discusses the main results. Section VII shows an application of the method to improve time seriesbased power forecasts. Section VIII draws the main conclusions.

\section{STATE-OF-THE-ART}

Among the several methods proposed in the literature for PV cell simulation, the single-diode five-parameter model 
proposed in [7] represents a good trade-off between having good accuracy and low computational complexity. While it is generally used to compute the $i-v$ curves of the PV cell, starting from sensed irradiance and cell temperature, we focus here on the reverse approach that uses measurements of DC electrical quantities ( $v$ and $i$ ) and cell temperature $T$ as inputs. Some research works from the existing literature exploit the reverse cell model but with different aims and applications than the one here proposed. For instance, in $[\overline{8}]$ three mathematical models to estimate the irradiance by sensing $v, i$ and $T$ in different load conditions (including short and open circuit), are analysed and compared. The main drawback is that most of existing PV systems are not designed to disconnect and move to short circuit and open circuit conditions. Authors of [9] propose a globally convergent estimator of the irradiance starting from $v, i$ and $T$ measurements, assuming that the plant operates in MPPT mode and without extending the analysis to the power estimation. Finally, in [10] the reverse model approach is proposed to implement MPPT for a single panel by integrating irradiance measurements from a dedicated pyranometer for the identification phase only.

With respect to the existing literature, we apply model-based filtering to the problem of reconstructing MPPT conditions of an entire PV system, starting from a generic operating point, with the model identified from datasheet information only. Furthermore, we show how this can be applied to improve time series-based power forecasting tools.

\section{Problem Statement}

We consider a time series of historical measurements of the DC voltage, current and cell temperature of a PV installation, which is possibly operating in curtailment regime. The problem is given by reconstructing the sequence of power production as if the plant was operating in MPPT mode $\AA^{1}$. We do this by developing a dedicated filter based on the well known fiveparameter model of a PV cell, and integrating measurements of the cell temperature $T$, DC voltage $v$ and DC current $i$ as measured on the PV module rear ${ }^{2}$ and converter DC bus, respectively. As an example of the application of the proposed approach, we show how it can be used to pre-filter the training time series of data-driven PV production forecasting models (which learn from historical power measurements) to improve their performance. The application is sketched in Fig. 1.

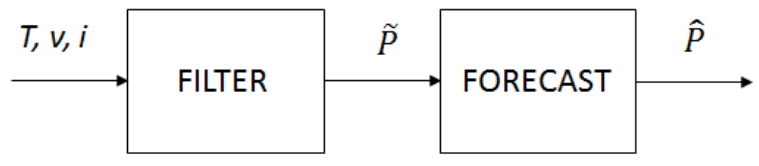

Fig. 1. Application of the proposed strategy. We indicate with $\tilde{P}$ the estimated maximum power and with $\hat{P}$ the forecasted one.

\footnotetext{
${ }^{1}$ We disregard here the effect of measurements uncertainties on the maximum power estimation. Work is ongoing to include a dedicated process capable of filtering measurement noise and eliminate measurement errors.

${ }^{2} \mathrm{We}$ hereby assume that the cell temperature is close enough to the module rear one, and that its value is uniform across the whole PV system.
}

\section{Methods}

This section describes the main components of the proposed filter. First, we introduce the five-parameter model of the PV cell, and the associated identification problem to determine the unknown parameters. Second, building up the five-parameter model, we derive the analytical method to estimate the solar irradiance incident on the panels, then used to reconstruct the maximum power point.

\section{A. PV Modeling}

The five-parameter model is a non-linear electric circuit model (shown in Fig. 2) useful to determine the current delivery capability of a PV cell as a function of the terminal DC voltage and the value of five parameters. Its extension to

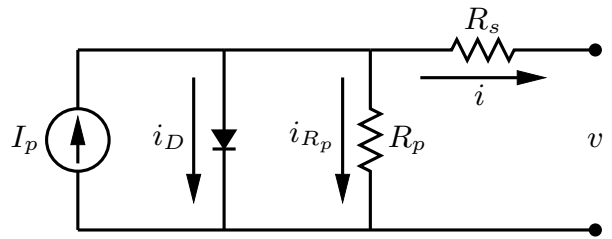

Fig. 2. The five-parameter circuital model for a PV cell.

a PV module composed of $n_{s}$ cells in series and $n_{p}$ in parallel is:

$$
i=I_{p} n_{p}-i_{D} n_{p}\left[\exp \left(q \frac{v+R_{s} i \frac{n_{s}}{n_{p}}}{n_{r} k T n_{s}}\right)-1\right]-\frac{v+R_{s} \frac{n_{s}}{n_{p}}}{R_{p} \frac{n_{s}}{n_{p}}}
$$

where $i$ is the DC current, $v$ DC voltage, $n_{r}$ diode ideality factor, $k$ Boltzmann constant, $q$ electron charge, $T$ cell temperature, $R_{s}$ and $R_{p}$ are the series and shunt resistances, $I_{p}$ and $i_{d}$ the light and saturation currents, respectively. The five unknown parameters of the model $R_{s}, R_{p}, I_{p}, i_{d}, n_{r}$ are first computed at reference conditions. Standard Test Conditions (STC) are temperature $T^{*}=25^{\circ} \mathrm{C}$ and irradiance $S^{*}=$ $1000 \mathrm{~W} \mathrm{~m}^{-2}$ and are here denoted with $\left(^{*}\right)$. The extraction of the five parameters at STC needs the following datasheet information: the open circuit voltage $\left(v_{O C}^{*}\right)$, the short circuit current $\left(i_{S C}^{*}\right)$, the voltage and the current at the maximum power $\left(v_{M P}^{*}\right.$ and $\left.i_{M P}^{*}\right)$, all at reference conditions, the absolute temperature coefficients of the open circuit voltage $(\beta)$ and of the short circuit current $(\alpha)$. In our case, we use the reduced form of the model, proposed in [11], that guarantees to find a physically feasible solution of the problem at first launch. The model is reduced from five to two parameters $\left(R_{s}\right.$ and $\left.n_{r}\right)$ and solved using the fsolve function in Matlab. The parameters computed at STC need to be updated for different irradiance 
and temperature conditions using the following equations:

$$
\begin{aligned}
R_{s} & =R_{s}^{*} \\
R_{p} & =R_{p}^{*} \frac{S^{*}}{S} \\
I_{p} & =\left(I_{p}^{*}+\alpha\left(T-T^{*}\right)\right) \frac{S}{S^{*}} \\
n_{r} & =n_{r}^{*} \\
i_{D} & =i_{D}^{*}\left[T / T^{*}\right]^{3} \exp \left(E_{g}^{*} / k T^{*}-E_{g} / k T\right) \\
E_{g} & =1.17-4.73 * 10^{-4} \frac{T^{2}}{T+636},
\end{aligned}
$$

where $E_{g}^{*}$ is the band gap energy at $T^{*}$, in $\mathrm{eV}$.

\section{B. Maximum Power Estimation}

As described in Section III several approaches have been proposed to compute the maximum power, starting from the physical model of the PV system. In the here proposed formulation, the irradiance is calculated analytically by substituting equations (2)-(6) into (1) and solving for $\tilde{S}$. Formally, it is:

$$
\tilde{S}=\frac{i+i_{D}^{*} n_{p}\left[T / T^{*}\right]^{3} \exp \left(E_{g}^{*} / k T^{*}-E_{g} / k T\right)\left[\exp \left(q \frac{v+R_{S}^{*} i n_{s} / n_{p}}{n_{r} k T n_{s}}\right)-1\right]}{\frac{1}{S^{*}}\left[n_{p}\left(I_{p}^{*}+\alpha\left(T-T^{*}\right)\right)-\frac{v+R_{S}^{*} i n_{s} / n_{p}}{R_{p}^{*} n_{s} / n_{p}}\right]}
$$

where $v, i$ and $T$ are measurable quantities. The estimated irradiance $\tilde{S}$ is then used, together with $T$, to compute the DC output power according to the following procedure:

- as defined in [12], the open circuit voltage $v_{O C}$ is:

$$
v_{O C}=v_{O C}^{*}\left(1+\beta\left(T-T^{*}\right)\right)+V_{t} n_{r} n_{s} \ln \left(\frac{\tilde{S}}{S^{*}}\right)
$$

where $V_{t}=k T / q$ is the thermal voltage;

- we compute the DC current for different DC voltage values by using Eq. (1), where $v$ varies between 0 and $v_{O C}$, this allowing to determine the $i$ - $v$ curve;

- we extract from the $i$ - $v$ curve the maximum power point for the PV module (maximum product $i \cdot v$ ). Then, assuming that each module has the same behaviour, we multiply the obtained value by the number of modules to get the system DC maximum power estimation.

\section{EXPERIMENTAL SETUP}

The experimental setup is located at the DESL laboratory at EPFL (GPS coordinates $46.518 \mathrm{~N}, 6.565 \mathrm{E}$ ) and consists of 4 identical strings with 14 ECSOLAR $255 \mathrm{~W}$ Polycrystalline PV modules each, connected in series. The strings are connected to two three-phase DC/AC converter equipped with a DC/DC converter that can operate in MPPT mode or under external control, i.e. it feeds electricity into the grid according to a specific active power external request. Two strings go to the first converter (here denoted as $\mathrm{C} 1$ ) and the other two to the second one (C2). The scheme is shown in Fig. 3. The two converters are of the same commercial model,independently controlled and connected to the same AC bus. This allows validating the method during curtailment regimes (operated in $\mathrm{C} 1$ ), by leaving $\mathrm{C} 2$ working in MPPT for the whole time and using its delivered power as the true maximum power value. DC currents are measured with LEM LF 205-S current transducers while the voltages are measured with a LEM-CV 3-100 voltage transducers. The temperature is measured using a TSic303 sensor installed on the rear surface of a PV panel, assuming the module temperature is close enough to the cell one, that is more difficult to measure. All the measurements are sampled with $10 \mathrm{sec}$ resolution. Only daylight data are selected for the analysis, which is assumed for solar elevation angles larger than $3^{\circ}$.

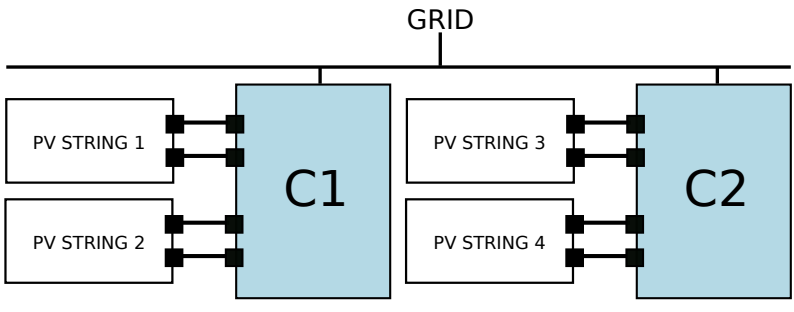

Fig. 3. Scheme of the experimental setup.

\section{EXPERIMENTAL RESULTS}

In this section, the maximum power estimated by the proposed approach is compared to the true maximum power value, which is assumed known from measurements as described in Section V The comparison is carried out for a clear-sky and a partly cloudy day. For each day we consider two distinct cases, which are denoted by MPPT Conditions and Curtailed Conditions. The former refers to periods when the converter operates in MPPT mode, while the latter to when it operates in curtailment regime. This is with the specific objective of validating the proposed method for a generic system working point, in other words without any a priori assumption on the fact if the plant is tracking the maximum power point or not.

To assess estimation performance, we use the relative Root Mean Squared Error (rRMSE) and the maximum error Err $_{\max }$ :

$$
\begin{aligned}
& \mathrm{rRMSE}=\frac{1}{\bar{P}} \sqrt{\frac{\sum_{t=1}^{m}\left(\tilde{P}_{t}-P_{t}\right)}{m}} \\
& \operatorname{Err}_{\max }=\max \left\{\left|\tilde{P}_{t}-P_{t}\right|, t=1, \ldots, m\right\}
\end{aligned}
$$

where $m$ is the number of considered estimations, $P$ the true maximum power value of the plant (provided by the converter working in MPPT mode), $\tilde{P}$ the estimated maximum power value, and $\bar{P}$ the average power in the considered period.

\section{A. MPPT Conditions}

The comparison between the true and estimated maximum power point values when the plant operates in MPPT mode is shown figures $4 \mathrm{a}$ and $5 \mathrm{a}$ for a clear-sky and partly cloudy day, respectively. Details are extracted in figures $4 b$ and $5 b$ to allow better visualization. Table I] summarizes the estimation performance metrics and shows a rRMSE error below 3\% level and a maximum error below $50 \mathrm{~W}$, for a rated power of the PV system of $7.14 \mathrm{~kW}$. 


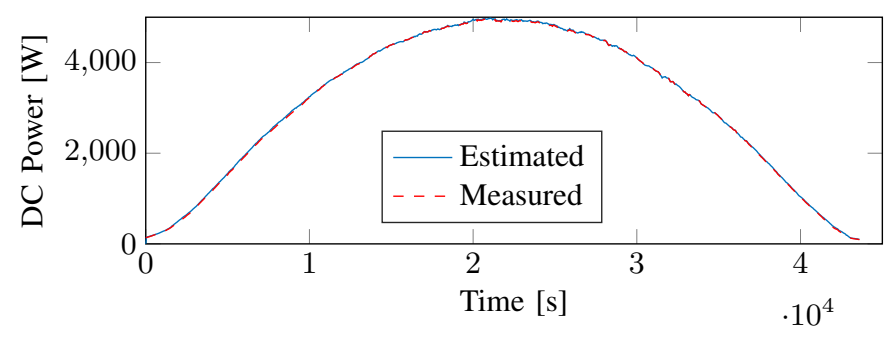

(a) MPPT Clear-sky Day

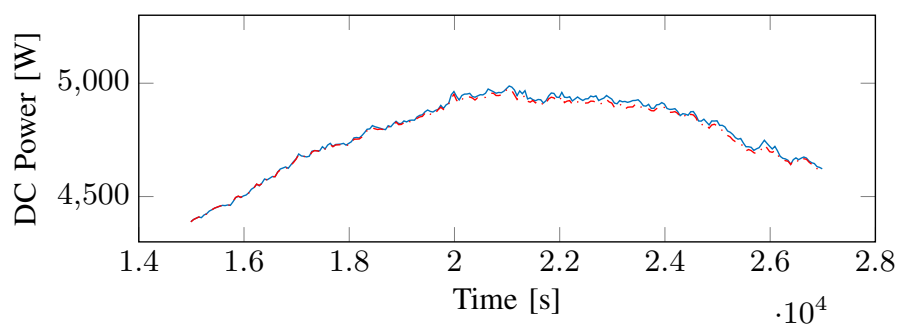

(b) MPPT Clear-sky Day-Detailed

Fig. 4. The true maximum power (from C2) is compared with the estimated one, considering a clear-sky day. The system works in MPPT conditions.

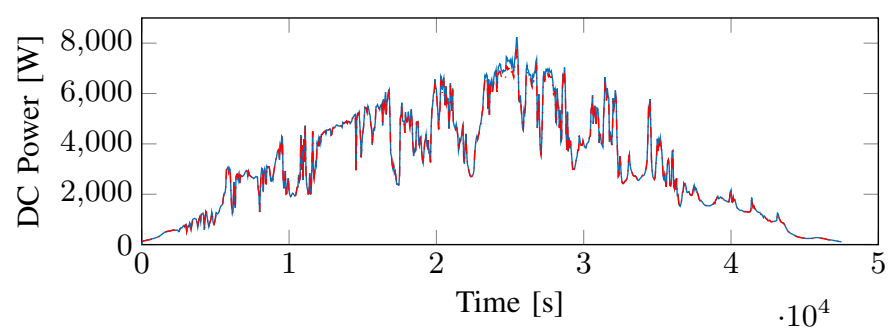

(a) MPPT Partly Cloudy

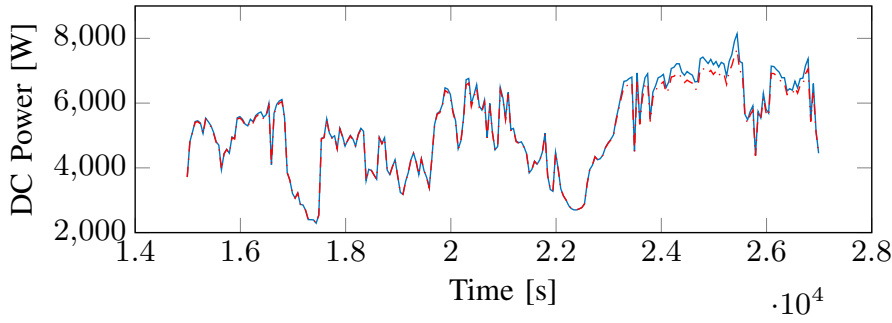

(b) MPPT Partly Cloudy Day-Detailed

Fig. 5. The true maximum power (from C2) is compared with the estimated one, considering a partly cloudy day. The system works in MPPT conditions.

TABLE I

RRMSE AND MAXIMUM ABSOLUTE ERROR IN MPPT CONDITIONS.

\begin{tabular}{|c|c|c|}
\hline & rRMSE [\%] & Err $_{\max }[\mathrm{W}]$ \\
\hline Clear-Sky & 0.51 & 45 \\
\hline Partly Cloudy & 2.35 & 37 \\
\hline
\end{tabular}

\section{B. Curtailed Conditions}

The analysis shown above for the MPPT case is here repeated considering the system in curtailment regime. Recalling from Section V, the output power of converter $\mathrm{C} 1$ is curtailed (shown in Fig.6for the two selected days), while the measured power of converter $\mathrm{C} 2$ is used as the true value for comparison. Estimations and true values are shown in figures $7 \mathrm{a}$ and $8 \mathrm{a}$ for two selected days, while a more detailed visualisation is shown in figures $7 \mathrm{~b}$ and $8 \mathrm{~b}$. The estimation performance metrics are summarized in Table II. We can state that the method is able to reconstruct the maximum power with small rRMSE (5$7 \%$ ) even when curtailment is adopted. However, performance of the maximum power point estimation is worse for the curtailment regimes than MPPT mode (especially in terms of maximum absolute error). This is explained by the larger sensitivity of the estimation with respect to the temperature when the system operates closer to open circuit conditions. This is clarified by Fig. 9, where two cases are considered: i) the DC voltage and current are fixed at their maximum point (MP) in STC conditions, and ii) the DC voltage and current are fixed at their open circuit (OC) point in STC conditions. From Fig. 9, it is well visible the high impact of temperature measurement errors on the estimated irradiance when closer to the open circuit point.
TABLE II

RRMSE AND MAXIMUM AbSOLUTE ERror In CURTAILEd Conditions.

\begin{tabular}{|c|c|c|}
\hline & rRMSE [\%] & Err $_{\max }[\mathrm{W}]$ \\
\hline Clear-Sky & 5.4 & 452 \\
\hline Partly Cloudy & 6.5 & 320 \\
\hline
\end{tabular}

\section{APPLICATION}

As a main application of the method, we show how it can be used to pre-filter historical power measurements and improve forecasting performance of data-driven prediction tools for PV generation. We select two artificial intelligencebased forecasting methods and compare two cases:

- Direct forecasting $(D F)$. The algorithm is trained with a time series composed of raw past power measurements where it is implicitly assumed that the system under consideration operates in MPPT mode.

- Filtered direct forecasting $(F D F)$. The algorithm is trained with pre-filtered power measurements according to proposed method using measurements of $v, i$ and $T$.

We use two datasets at $5 \mathrm{~min}$ resolution, each consisting of 30 days for the training and 5 for testing.In the first dataset, the system entirely operates in MPPT mode. In the latter, it is curtailed according to a random pattern for 12 training days and 2 testing days; this is done by controlling the PV converter active power set-point, while it is left in MPPT mode for the remaining days. The forecast horizon is $5 \mathrm{~min}$.

\section{A. Selected Forecast Methods}

In this Section, we present the two selected forecasting methods. The first is for point predictions and consists in 


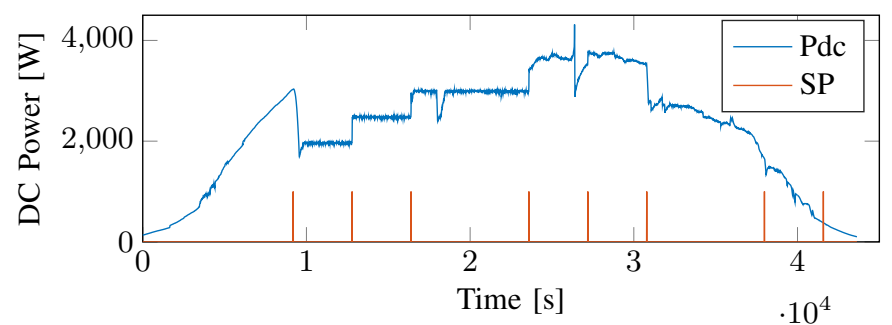

(a) Curtailed Clear-sky Day

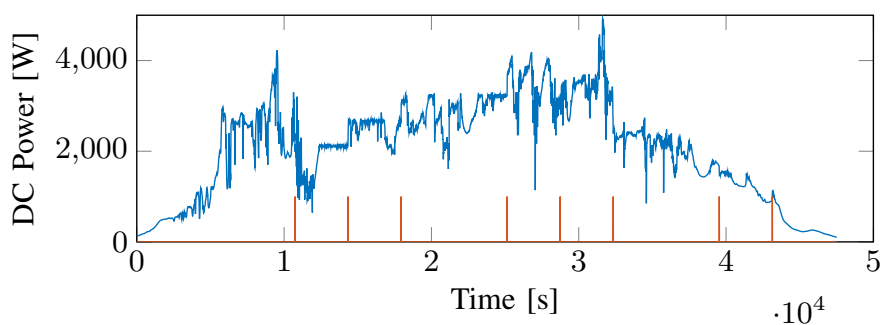

(b) Curtailed Partly Cloudy

Fig. 6. The true measured power of $\mathrm{C} 1$ is shown, for which the power output is curtailed. The red spikes individuate a set-point change.

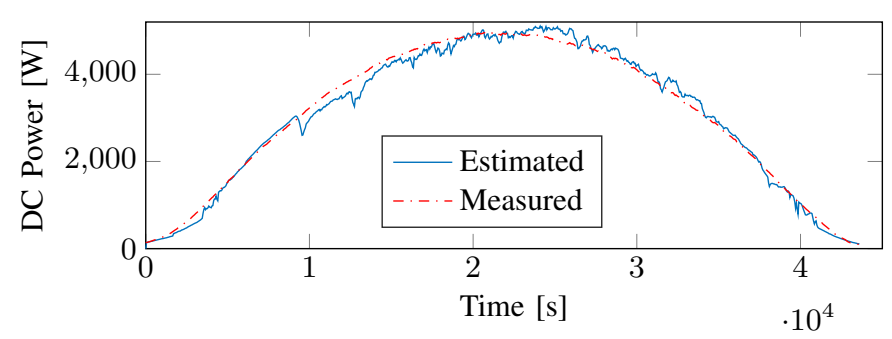

(a) Curtailed Clear-sky Day

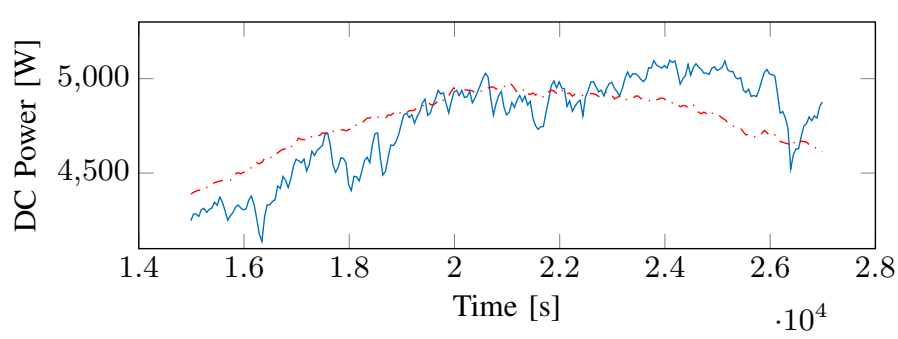

(b) Curtailed-Clear-sky Day-Detailed

Fig. 7. The true maximum power (from $\mathrm{C} 2$ ) is compared with the estimated one, considering a clear-sky day and power curtailment.

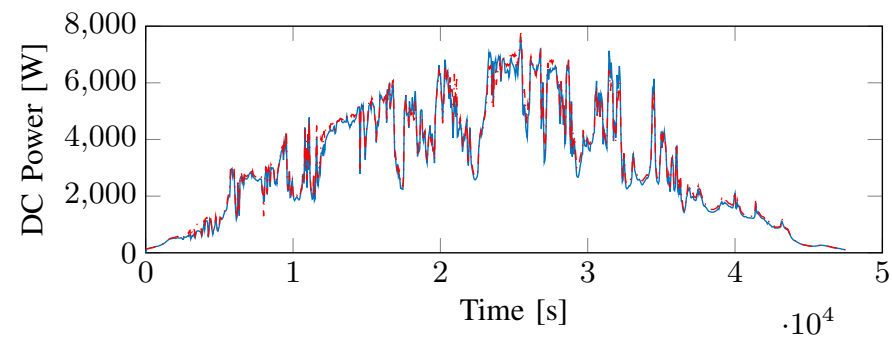

(a) Curtailed Partly Cloudy

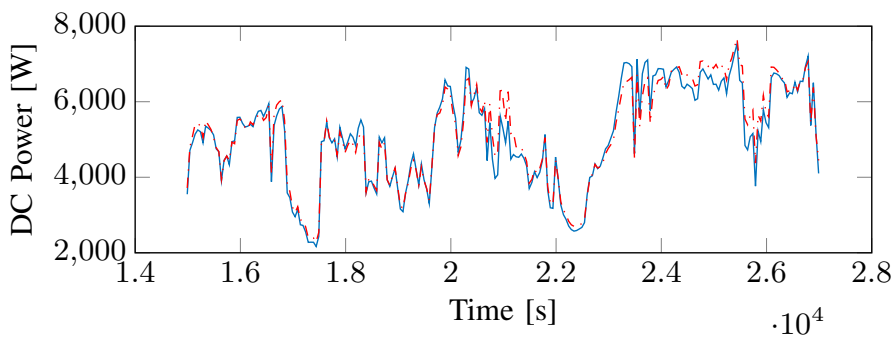

(b) Curtailed Partly Cloudy-Detailed

Fig. 8. The true maximum power (from $\mathrm{C} 2$ ) is compared with the estimated one, considering a partly cloudy day and power curtailment.

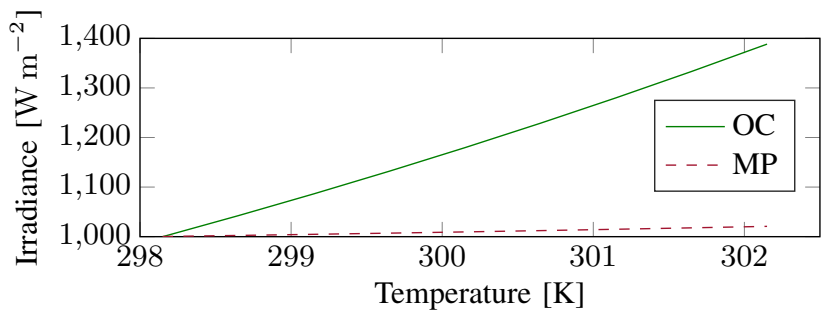

Fig. 9. The estimated irradiance is plotted as a function of the cell temperature for fixed $v$ and $i$. The dashed line is obtained by fixing $v=v_{m p}^{*}$ and $i=i_{m p}^{*}$, i.e. in MPPT STC conditions, while the continuous line refers to open circuit (OC) conditions, i.e. $v=v_{O C}^{*}$ and $i \approx 0$.

an artificial neural network (ANN), with 10 neurons in the network's hidden layer, trained by applying the LevenbergMarquardt back-propagation algorithm, [13], with Matlab. The second method is for prediction intervals (PIs, defined as the range $\left(P^{\uparrow}, P^{\downarrow}\right)$ where a realization is expected to fall with a given confidence level) and it was proposed in [14] for irradiance forecast. The method is here re-adapted to directly forecast the DC power output. It consists in, first, clustering historical observations of the power production according to the value of selected influential variables. In particular, we consider a training dataset of historical differentiated power measurements $d P_{1}, \ldots, d P_{N}$, from which we extract the i) the normalized average power value on a mobile window, and ii) the normalized power variability, as it is formulated in [14]. Then, we use the $k$-mean algorithm to cluster the training set. During the on-line computation, when a new power measurement $\left(P_{t}\right)$ is available, the influential variables are calculated in real-time and used to select the cluster that resembles at most the actual conditions. The most similar cluster is therefore used to compute the PI by calculating the symmetric quantiles ( $q^{\uparrow}$ and $q^{\downarrow}$ ) according to a given confidence level (i.e. the cluster is considered as an empirical conditional probability function of future realizations). PIs are calculated by summing the current measurement to the upper 
and lower quantiles of the differentiated time series:

$$
\begin{aligned}
& P_{t+1 \mid t}^{\uparrow}=P_{t}+q^{\uparrow}, \\
& P_{t+1 \mid t}^{\downarrow}=P_{t}+q^{\downarrow},
\end{aligned}
$$

It is important to highlight that we are not interested here in assessing the skills of the forecasting methods, rather showing the advantage introduced by the proposed pre-filtering approach in the forecasting chain.

\section{B. Forecast improvement}

The metric used to evaluated point predictions performance is the mean absolute error (MAE):

$$
M A E=\frac{1}{N} \sum_{t=1}^{N}\left(\hat{P}_{t+1 \mid t}-P_{t+1}\right)
$$

where $\hat{P}$ is the one-step-ahead prediction, $P$ is the realization, and $N$ is the number of measurements in the testing dataset. For the case of PIs, we use standardized metrics from the literature, see [15]. The first is the PI coverage probability (PICP, which counts the number of realization inside the PI):

$$
\mathrm{PICP}=\frac{1}{N} \sum_{t=1}^{N} c_{t} \quad c_{t}= \begin{cases}1, & \hat{P}_{t+1 \mid t}^{\downarrow} \leq P_{t+1} \leq \hat{P}_{t+1 \mid t}^{\uparrow} \\ 0, & \text { otherwise. }\end{cases}
$$

Then, to account for the fact that the wider the PI, the easier it is to have a realization falling inside it, we measure the prediction interval normalized width (PINAW):

$$
\text { PINAW }=\frac{1}{N P_{\text {max }}} \sum_{t=1}^{N}\left(\hat{P}_{t+1 \mid t}^{\uparrow}-\hat{P}_{t+1 \mid t}^{\downarrow}\right) .
$$

where $P_{\max }$ is the system rated power, in our case equal to $7.14 \mathrm{~kW}$. The target confidence level is set equal to $95 \%$. Results are summarized in Tables III and Tables IV for the point and PI forecast, respectively. When the system works in

TABLE III

ANN-MAE

\begin{tabular}{|c|c|c|}
\hline [W] & MPPT & Curtailed \\
\hline DF with ANN & 223 & 640 \\
\hline FDF with ANN & 213 & 295 \\
\hline
\end{tabular}

TABLE IV

K-MEAN-PICP/PINAW

\begin{tabular}{|c|c|c|}
\hline$[\%]$ & MPPT & Curtailed \\
\hline DF with $k$-mean & $96.6-18.2$ & $79-20.54$ \\
\hline FDF with $k$-mean & $96.6-18.2$ & $96.2-18.2$ \\
\hline
\end{tabular}

MPPT state, performance are not altered by the filter. However, it is clear the benefit introduced by the proposed technique when curtailment strategies are adopted. In this case, the DF produces large errors whilst our method has lower MAE for the ANN case and higher PICP with lower PINAW for the PIs $k$-mean-based forecasting method.

\section{CONCLUSION}

We have described a model-based technique to estimate the maximum power value of a PV production plant by using measurements of the DC voltage, DC current and the module temperature. It exhibits overall good estimation performance in different operating conditions when tested in a real experimental setup, in both clear-sky and partly cloudy conditions. Indeed, we obtained a maximum rRMSE below 7\%. It was found that estimation error is larger when operating closer to open-circuit conditions due to a large sensitivity with respect to temperature measurements. As an application, we show how the method can be applied to pre-filter historical PV production measurements prior to use them to train data-driven forecasting models. It was shown that its use is always beneficial to improve prediction performance. In particular, when historical measurements are in MPPT mode, filtering does not negatively affect performance, while it leads to a substantial improvement when historical data includes operation in non-MPPT mode.

\section{REFERENCES}

[1] E. Ela, M. Milligan, and B. Kirby, "Operating reserves and variable generation," Contract, vol. 303, pp. 275-3000, 2011.

[2] J. Antonanzas, N. Osorio, R. Escobar, R. Urraca, F. M. de Pison, and F. Antonanzas-Torres, "Review of photovoltaic power forecasting," Solar Energy, vol. 136, pp. 78 - 111, 2016.

[3] R. H. Inman, H. T. Pedro, and C. F. Coimbra, "Solar forecasting methods for renewable energy integration," Progress in Energy and Combustion Science, vol. 39, no. 6, pp. 535 - 576, 2013.

[4] H. T. Pedro and C. F. Coimbra, "Assessment of forecasting techniques for solar power production with no exogenous inputs," Solar Energy, vol. 86, no. 7, pp. 2017 - 2028, 2012.

[5] F. Golestaneh, P. Pinson, and H. Gooi, "Very short-term nonparametric probabilistic forecasting of renewable energy generation with application to solar energy," 2016.

[6] R. Tonkoski, L. Lopes, and T. El-Fouly, "Droop-based active power curtailment for overvoltage prevention in grid connected pv inverters," in 2010 IEEE International Symposium on Industrial Electronics. IEEE, 2010, pp. 2388-2393.

[7] W. De Soto, S. Klein, and W. Beckman, "Improvement and validation of a model for photovoltaic array performance," Solar energy, vol. 80, no. 1, pp. 78-88, 2006.

[8] V. L. Vigni, D. L. Manna, E. R. Sanseverino, V. di Dio, P. Romano, P. di Buono, M. Pinto, R. Miceli, and C. Giaconia, "Proof of concept of an irradiance estimation system for reconfigurable photovoltaic arrays," Energies, vol. 8, no. 7, pp. 6641-6657, 2015.

[9] M. Carrasco, F. Mancilla-David, and R. Ortega, "An estimator of solar irradiance in photovoltaic arrays with guaranteed stability properties," IEEE Transactions on Industrial Electronics, vol. 61, no. 7, pp. 33593366, 2014.

[10] L. Cristaldi, M. Faifer, M. Rossi, and S. Toscani, "An improved modelbased maximum power point tracker for photovoltaic panels," IEEE transactions on instrumentation and measurement, vol. 63, no. 1, pp. 63-71, 2014.

[11] A. Laudani, F. Mancilla-David, F. Riganti-Fulginei, and A. Salvini, "Reduced-form of the photovoltaic five-parameter model for efficient computation of parameters," Solar Energy, vol. 97, pp. 122 - 127, 2013.

[12] "IEC60891, Photovoltaic Devices. Procedures for Temperature and Irradiance Corrections to Measured IV Characteristics." IEC60891, 2010.

[13] M. T. Hagan and M. B. Menhaj, "Training feedforward networks with the marquardt algorithm," IEEE transactions on Neural Networks, vol. 5, no. 6, pp. 989-993, 1994.

[14] E. Scolari, F. Sossan, and M. Paolone, "Irradiance prediction intervals for $\{\mathrm{PV}\}$ stochastic generation in microgrid applications," Solar Energy, vol. 139 , pp. 116 - 129, 2016.

[15] A. Khosravi, S. Nahavandi, and D. Creighton, "Prediction intervals for short-term wind farm power generation forecasts," Sustainable Energy, IEEE Transactions on, vol. 4, no. 3, pp. 602-610, 2013. 